\title{
AN ELECTRO-HYDRAULIC SERVO MECHANISM WITH AN ANALOG ADAPTIVE CONTROLLER
}

\author{
Kazushi Sanada, Yoshiyasu Hagiwara, Ato Kitagawa \\ Dept. of Control Engineering \\ Faculty of Engineering \\ Tokyo Institute of Technology \\ Tokyo, Japan
}

\section{ABSTRACT}

In recent years, many studies on the adaptive control of an oilhydraulic servomechanism using a microcomputer have been done. A microcomputer has merits of flexibility of algorism and high computing accuracy. But the computing time of the discrete adaptive law is not short enough to control an oilhydraulic servomechanism in high frequency region. For control of an oilhydraulic servomechanism in higher frequency region, there are two method. One is a method of using a digital signal processor, and the other is a method of using an analog adaptive controller. The analog adaptive controller has a merit that its computing time is theoretically zero. In this study, the adaptive law was constructed in continuous time description and the adaptive controller was realized by an analog electronic circuit which was designed using a CAD program. And experiments were conducted to investigate the electrohydraulic servomechanism with the analog adaptive controller.

\section{KEYWORDS}

Electrohydraulic Servomechanism, Adaptive Control, CAD, Analog Adaptive Controller

\section{NOMENCLATURE}

\footnotetext{
$a \quad:$ linearized parameter

$a_{M}:$ reference model parameter

$b$ : linearized parameter

$b_{M}:$ reference model parameter
}

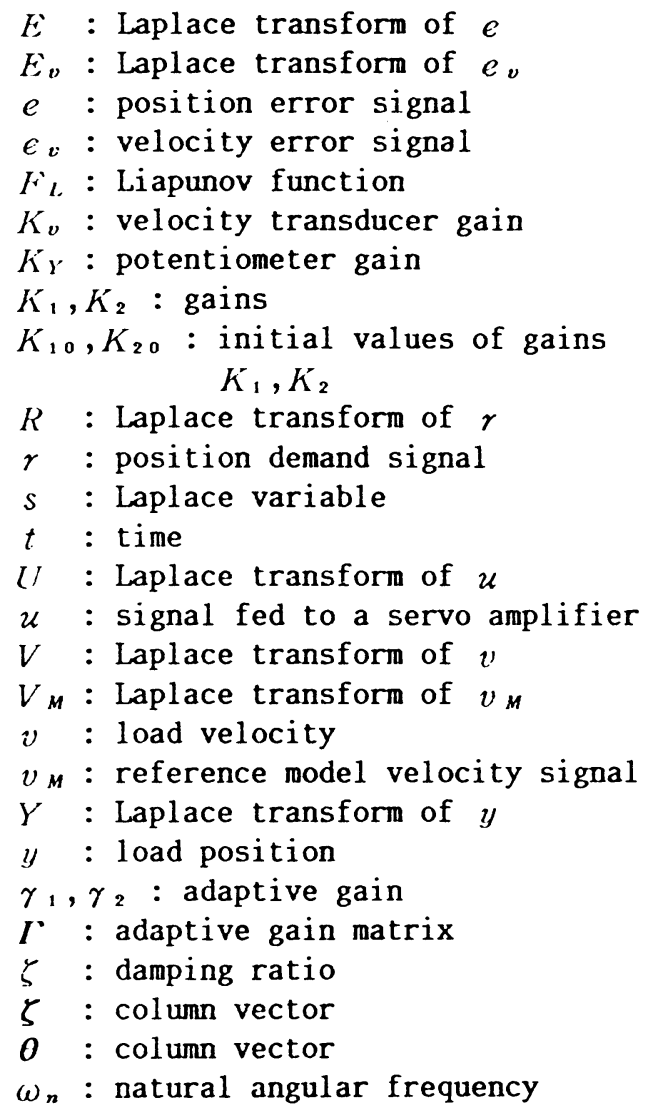

Superscript

$T:$ transposed matrix

-1 : inverse matrix

\section{INTRODUCTION}

In recent years, many studies on the adaptive control of an oilhydraulic servomechanism have been done $(1) \sim(4)$. In these studies, microcomputers were used as adaptive controllers. A microcomputer has 
206

merits of control algorism flexibility and high computing accuracy. For realizing an adaptive control system with a microcomputer, the adaptive control law must be constructed in discrete time description. Since the discrete-time adaptive control law contains many computations of the four rules of arithmetic, the calculation of one cycle of the control law using a microcomputer needs at least several milliseconds. For this computing time, sampling interval may not be designed short enough to control an oilhydraulic servomechanism in high frequency region and to realize a high-speed servo system.

There are two methods in order to realize a high speed adaptive system. One is a method of using a digital signal processor. By this method, computing time can be considerably shortened. The other is a method of using an analog adaptive controller. In the latter method, the adaptive control law is constructed in continuous time description and an adaptive controller is realized by an analog electronic circuit (5). In order to make the adaptive control more practical control method for an electrohydraulic servomechanism, it is necessary to investigate these two methods in detail. In this study, the latter method, that is the method of utilizing an analog adaptive controller, was investigated. Since an analog adaptive controller does not discretize continuous signals and can perform real time calculation of the adaptive law, the computing time of the analog adaptive controller is theoretically zero.

In this study, at first, the adaptive law was constructed in continuous time description. Secondly, a CAD program for an analog adaptive controller was programed. Using this CAD program, fundamental design data of the controller were obtained. At last, experiments were conducted by use of an oilhydraulic servomechanism with an analog adaptive controller realized by an analog electronic circuit.

\section{CONTINUOUS-TIME ADAPTIVE LAW}

In this chapter, the adaptive law is constructed in continuous time description. A schematic diagram of an oilhydraulic servomechanism is presented in Fig.2.1. The object of this servomechanism is to control position of a mass-load (1). Position and velocity of the load are detected by a potentiometer (4) and a velocity transducer (5). Using these signals, an adaptive controller (6) performs calculations of the control law. The output signal of the controller is fed to a servo amplifier.
Then a servo valve (3) controls oil flow and a symmetrical cylinder (2) moves a mass-load. In this investigation, it is assumed that the transfer characteristics between input signal fed to a servo amplifier and load velocity can be approximated by a first order lag system and transfer characteristics of this system change or are not known. Then the adaptive control theory is applied to the block of the first order lag system. Therefore the servo system becomes the position feedback system containing the adaptive control system for load velocity.

The transfer function between input signal fed to a servo amplifier and load velocity is written as

$$
l(s)=\begin{aligned}
& b \\
& s+a
\end{aligned}
$$

Adding a positive feedback compensation block of gain $K_{1}$ and an input signal compensation block of gain $K_{2}$ to the first order lag system Eq.(1), the block diagram of the compensated system becomes as shown in Fig.2.2. The input signal of the system is position error signal $e$. The transfer function of this system is written as

$$
V^{\prime}(s)=\frac{K_{2} b}{s+\left(a-K_{1} b\right) .}
$$

A reference model is presented as

$$
V_{M}(s)=\begin{gathered}
b_{M} \\
s+a_{M}
\end{gathered}
$$

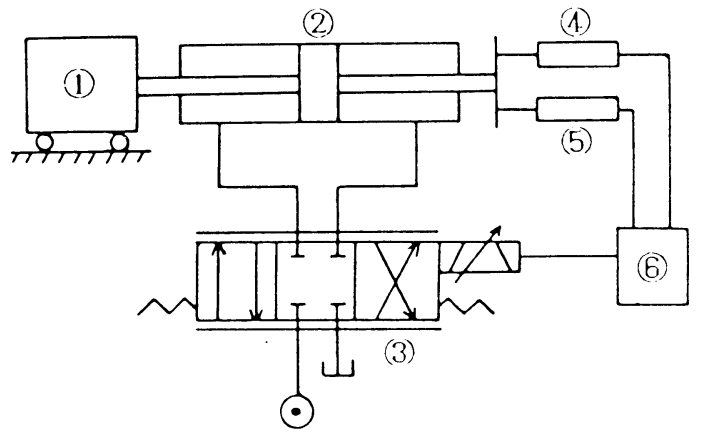

Fig.2.1 Schematic diagram of an electrohydraulic servomechanism

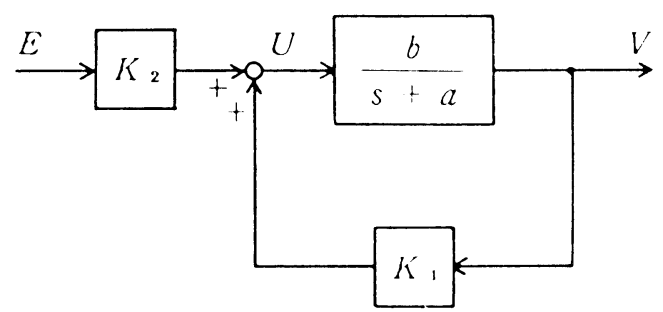

Fig.2.2 Block diagram of the compensated system 
Parameters $a$ and $b$ are linearized parameters of the plant. If these parameters are known and constant, gains $K_{1}$ and $K_{2}$ can be designed as follows so that load velocity can follow reference model velocity signal:

$$
K_{1}=\frac{a-a_{M}}{b}, \quad K_{2}=b_{M}^{b}
$$

But linearized parameters $a$ and $b$ of a real oilhydraulic servomechanism are not constant or unknown. Therefore the design of $K_{1}$ and $K_{2}$ according to Eq. (4) may not be carried out or may cause insufficient control results. In such a case, the adaptive control theory becomes a necessary and effective control method.

The adaptive control law is constructed in continuous time description. The control law becomes as follows (5), (7). The total block diagram of the adaptive control system is presented in Fig.2.3.

- position error

$$
c=r-k_{Y} y
$$

- velocity error

$$
\epsilon_{v}=v-v_{M}
$$

- signal fed to a servo amplifier

$$
u=K_{1} v+K_{2} e
$$

- adaptive laws of gains $K_{1}$ and $K_{2}$

$$
\begin{gathered}
K_{1}=-\left.\gamma_{1}\right|_{v_{0}} ^{t} v c_{v} \mathrm{~d} t+K_{10} \\
K_{2}=-\gamma_{2} \int_{0}^{t} c e_{v} \mathrm{~d} t+K_{20} \\
\left(\gamma_{1}, \gamma_{2}>0\right)
\end{gathered}
$$

It is demonstrated as follows that Eq. (8) and Eq. (9) are adaptive laws of gains $K_{1}$ and $K_{2}$.

After obtaining inverse Laplace transform of Eq. (2) and Eq. (3) and calculating velocity error $\mathcal{C}_{v}$, a differential equation for $e_{\nu}$ is obtained;

$$
\frac{\mathrm{d}}{\mathrm{dl}} c_{v}=-a_{M} e_{\nu}+b \theta^{T} \zeta \text {. }
$$

where

$$
\left\{\begin{array}{l}
0^{T}=\left[K_{1}-\frac{a-a_{M}}{b}, K_{2}-\frac{b_{M}}{b}\right] . \\
\zeta^{T}=[v, c] .
\end{array}\right.
$$

Differentiating Eq.(8) and Eq. (9), and arranging by use of Eq. (11), the following equation is obtained';

$$
\frac{\mathrm{d}}{\mathrm{d} \mathrm{\theta}} \boldsymbol{\theta}=-\Gamma \zeta e_{v} .
$$

$I$ is an adaptive gain matrix;

$$
\Gamma=\left[\begin{array}{ll}
\gamma_{1} & 0 \\
0 & \gamma_{2}
\end{array}\right]
$$

By the fact that the following function Eq. (14) is a Liapunov function, it is demonstrated that the simultaneous differential equation system Eq.(10) and Eq. (12) is a stable system.

$$
F_{L}=\frac{1}{2} e_{v}^{2}+\frac{1}{2} b \theta^{T} \boldsymbol{I}^{-1} \boldsymbol{\theta}
$$

Since the simultaneous differential equation system Eq.(10) and Eq. (12) is stable, one of state variables, velocity error $\epsilon_{v}$, converges zero as time approaches infinity. Therefore load velocity $v$ can follow reference model velocity signal $v_{M}$. It has been demonstrated that Eq. (8) and Eq. (9) are adaptive laws of gains $K_{1}$ and $\mathrm{K}_{2}$.

As evident from Eq. (5) (9), the adaptive law constructed in continuous time description contains addition, subtraction, multiplication and integral. The adaptive

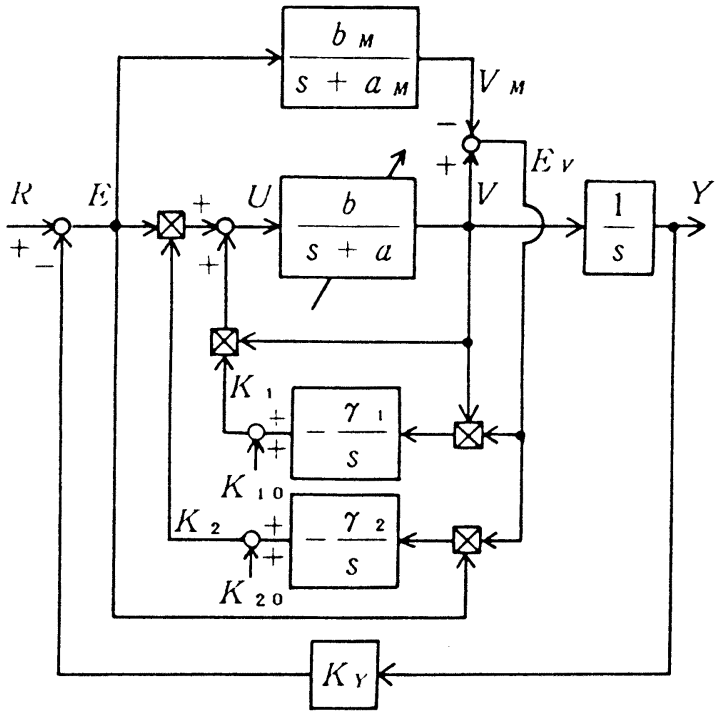

Fig.2.3 Block diagram of the adaptive control system

1 Eq. (12) holds when $a$ and $b$ are time invariant. Linearized parameters $a$ and $b$ of the system treated in this study are tine variant parameters. But, when computing speed of the control law is much faster than the speed of change of $a$ and $b$, Eq. (12) is considered to hold. 
law constructed in discrete time description contains division $(1) \sim(5)$. On the contrary, the continuous-time adaptive law dose not contain division. This becomes a considerably favorable merit for designing an adaptive controller.

\section{DESIGN OF AN ANALOG ADAPTIVE CONTROLLER}

In the above chapter, the adaptive law
was constructed in continuous time description. In this chapter, an analog adaptive controller which is realized by an analog electronic circuit is designed. In design of an analog adaptive controller, the design process was divided into two processes. In the first process, from simulations based on the block diagram of the adaptive control system presented in Fig.2.3, reference model parameters $a_{M}$ and $b_{M}$ and adaptive gains $\gamma_{1}$ and $\gamma_{2}$ were determined. Simulations were performed considering flow characteristics of a servo valve, oil compressibility and satulation characteristics of a servo amplifier etc.. Transient responses were obtained from numerical calculations of equations of motion of a servomechanism by the RungeKutta method. In the second process, simulations were performed based on an analog electronic circuit of an adaptive controller. Resistances and condensers were designed.

In order to design an analog adaptive controller, simulations must be done repeatedly for various parameters of the controller. In this study, for simulations and design, a CAD program of an analog adaptive controller was programed on NEC PC9801VX by use of a language of Turbo Pascal. In this CAD program, input and change of parameters of a servomechanism and an analog electronic circuit are possible and two kinds of the above mentioned simulations can be performed. And also frequency responses of the servo system only with feedback control, without adaptive control, can be calculated. Frequency responses of the feedback control system are useful data for determining reference model parameters.

Before starting design processes, specifications of the servo system were inputted to the CAD program. As presented in Fig.2.1, an oil-hydraulic servomechanism to be treated in this study consisted of a symmetrical cylinder, a servo valve and a mass-load. A symmetrical cylinder was $6.4 \times 10^{-4} \mathrm{~m}^{2}$ in pressure exerted area and 150 $\mathrm{mm}$ in piston stroke. A servo valve was a model 3F-30L (Tokyo Precision Instruments Co.,Ltd.). Specified supply pressure of the servo valve was $14 \mathrm{MPa}$, specified flow rate was $5 \times 10^{-4} \mathrm{~m}^{3} / \mathrm{s}$ and specified input current was $30 \mathrm{~mA}$. As a mass-load, sevral iron boards which were bolted up and attached to a cylinder rod were used. Four ball bearings were used as wheels of the massload. Mass of the load was able to be selected from about $20 \mathrm{~kg}$ to $60 \mathrm{~kg}$ according to number of iron boards bolted up. Gain of a potentiometer was $300 \mathrm{~V} / \mathrm{m}$ and gain of a velocity transducer was $11.8 \mathrm{~V} /(\mathrm{m} / \mathrm{s})$. Supply pressure was set at $5 \mathrm{MPa}$ by a relief valve.

In the first process of design, reference model parameters and adaptive gains were determined as follows. When the adaptive control is perfectly performed and load velocity completely follows reference model velocity signal, transfer characteristics of the adaptive control system perfectly match those of the reference model. Then a block diagram of the servo system on such a condition can be written as Fig.3.1. Refernce model can be designed based on the block diagram Fig.3.1. The transfer function of the closed loop system presented in Fig.3.1 is written as

$$
\begin{aligned}
& \frac{Y}{R}(s)=\frac{1}{K_{Y}} \cdot \frac{K_{Y} b_{M}}{s^{2}+a_{M} s+K_{Y} b_{M}} \\
& =\frac{1}{K_{Y}} \cdot \frac{\omega_{n}^{2}}{s^{2}+2 \zeta \omega_{n} s+\omega_{n}^{2}} .
\end{aligned}
$$

where

$$
\omega_{n}=v^{\prime} K_{Y} b_{M}, \quad \zeta=\frac{a_{M}}{2 v^{\prime} K_{Y} b_{M}} .
$$

Arranging Eq. (16), $a_{M}$ and $b_{M}$ can be written as

$$
a_{M}=2 \zeta \omega_{n}, \quad b_{M}=\begin{aligned}
& \omega_{n}^{2} \\
& k_{Y} .
\end{aligned}
$$

Using Eq.(17), reference model parameters $a_{M}$ and $b_{M}$ can be determined from natural angular frequency $\omega_{n}$ and damping ratio $\zeta$ which are specified by servo system designers. To specify natural angular frequency and damping ratio, transfer characteristics of the position feedback control system without the adaptive control are able to be utilized as reference

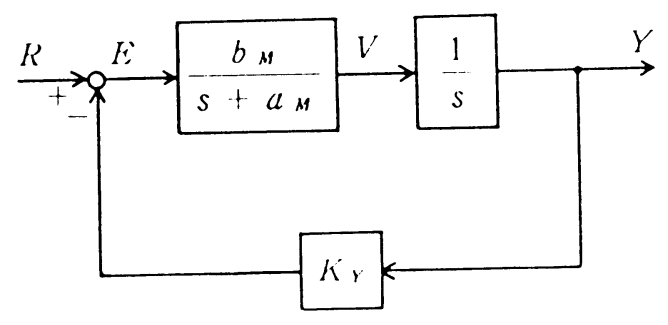

Fig.3.1 Block diagram for design of reference model 
data. Frequency responses were calculated using a linearized mathematical model of the servo system. Approximating frequency responses by that of a second order lag system, it was estimated that natural angular frequency was about $80 \mathrm{rad} / \mathrm{s}(13 \mathrm{~Hz})$ and damping ratio was about 0.8 . Desired transfer characteristics were designed better. In this study, desired natural angular freqeuency $\omega_{n}$ was designed as $170 \mathrm{rad} / \mathrm{s}(27 \mathrm{~Hz})$ and desired damping ratio $\zeta$ was designed as 0.5 . Substituting these values to Eq. (17), refernce model parameters were obtained;

$$
a_{M}=228.0 \mathrm{~s}^{-1}, \quad b_{M}=120.3 \mathrm{~m} /\left(\mathrm{V} \cdot \mathrm{s}^{2}\right) .
$$

Using these values of reference model parameters, simulations based on the adaptive control system presented in Fig.2.3 were conducted. From simulated results for various adaptive gains, it was determined that $\gamma_{1}=0.01$ and $\gamma_{2}=10.0$.

In the second process of design, an analog electronic circuit was designed. In Fig.3.2, an analog electronic circuit which realizes the adaptive law is presented. Triangle symbols in Fig.3.2 represent operational amplifiers (LM318) and pentagonal symbols represent analog multipliers (ICL8013). In this figure, some values of resistences and condensers are specified. Other resistances whose values are not specified have a value of $10 \mathrm{k} \Omega$. A position demand signal is fed to a terminal (1) and a position signal of a load detected by a potentiometer is fed to a terminal (2). A velocity signal detected by a velocity transducer is fed to a terminal (3). A signal (4) is the output signal of a low-pass filter which represents transfer characteristics of a reference model. Using high accurate variable resistances, reference model parameters are able to be adjusted. A siganl (5) corresponds to a velocity error signal. Signals (6) and (7) are outputs of adaptive mechanisms consisted of multipliers, integraters ( A and B ) and variable gain amplifiers. These blocks correspond to adaptive laws of gains $K_{1}$ and $K_{2}$ (Eq. (8) and Eq. (9)). Adaptive gains are able to be turned by variable resistences. Signal (8) is fed to a servo amplifier.

Generally, voltage of a signal in an analog electronic circuit is limitted by about supply voltage. The limit voltage of the circuit used was $\pm 13 \mathrm{~V}$. And the limit voltage of input and output signal of an analog multiplier was $\pm 10 \mathrm{~V}$. Resistances and condensers must be desined so that signal voltage should not exceed these limit voltages. In Fig.3.3, simulated results of transient responses of the adaptive control system with the analog adaptive controller are presented. It was confirmed that any signal did not exceed limit voltages. Since the signal corresponding to velocity error almost converged to zero, it has been cleared that the adaptive control was almost completely performed.

Computing time of an analog electronic circuit is theoretically zero. But, when designing a real circuit, it is necessary to examine frequency band width of an operational amplifier circuit and an analog multiplier. Band width of an integral circuit used for experiments was $0.08 \sim 320 \mathrm{~Hz}$. That of an addition or subtraction circuit was $D C \sim 15 \mathrm{MHz}$. And that of an multiplier was $\mathrm{DC} \sim 10 \mathrm{kHz}$. Comparing with frequency region of transient responses of the servo system, these band width of

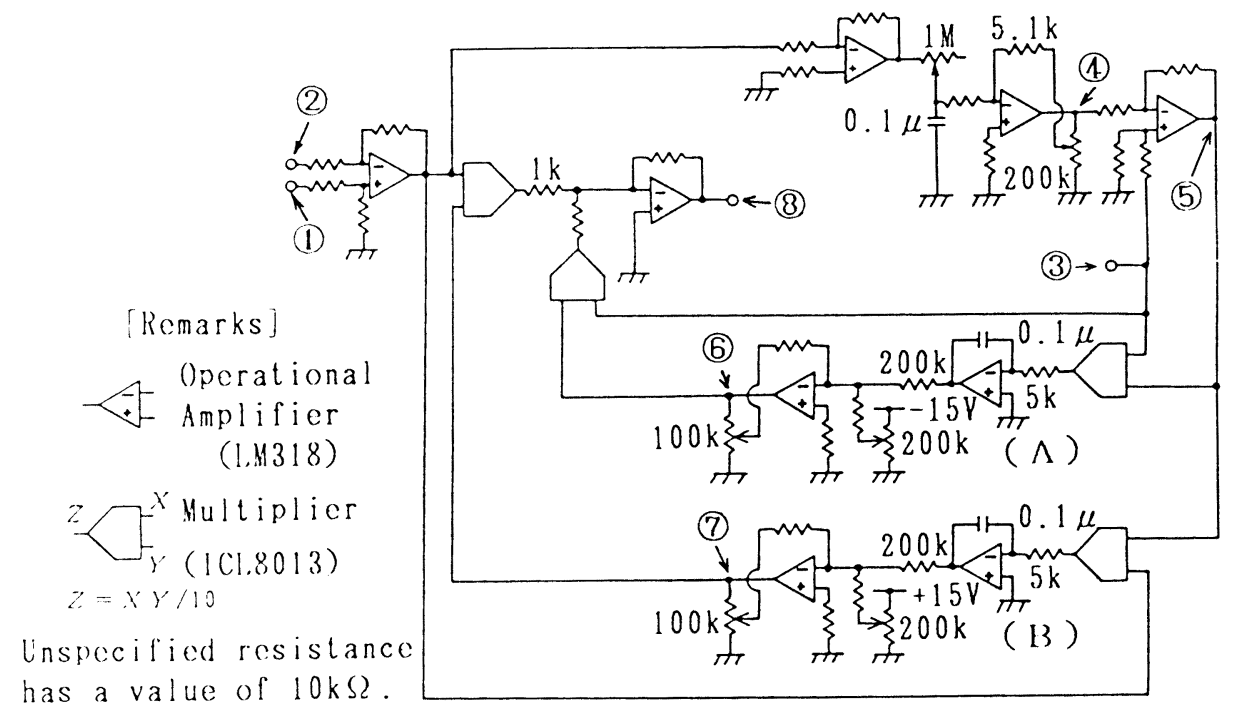

Fig.3.2 An analog electronic circuit of the analog adaptive controller 
210

analog circuits were wide enough.

\section{EXPERIMENTS}

In this chapter, experiments on an oilhydraulic servo system with the analog adaptive controller are described. The servomechanism used for experiments has been mentioned in the above chapter.

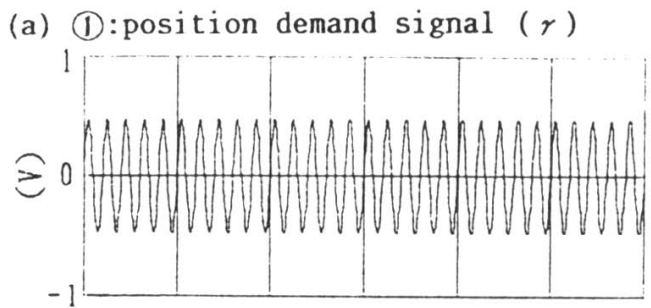

(b) (2):load position signal ( $\left.K_{Y} y\right)$

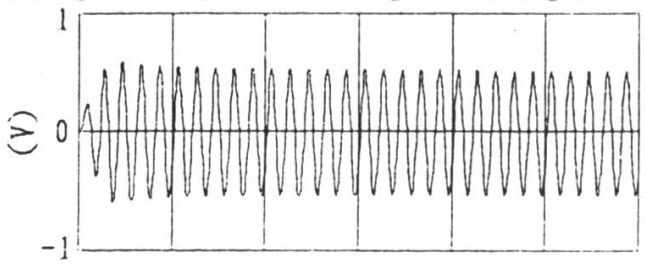

(c) (3): load velocity signal $\left(K_{v} v\right)$

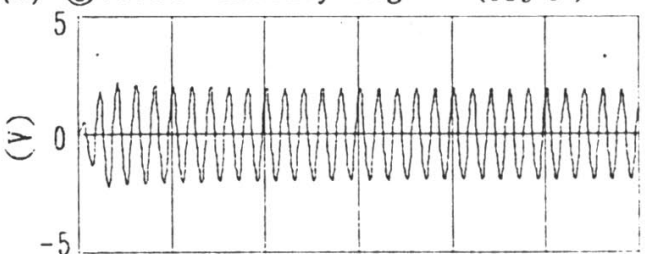

(d) (4): reference model velocity signal

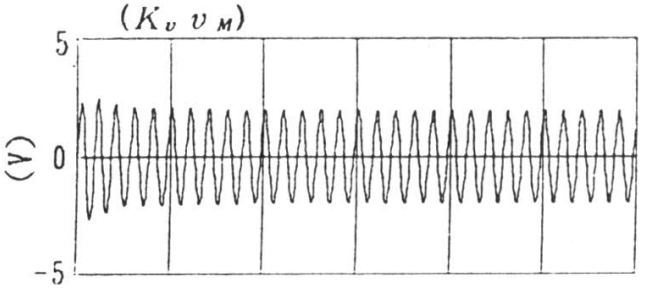

(e) (5):velocity error signal $\left(K_{n} \epsilon_{n}\right)$

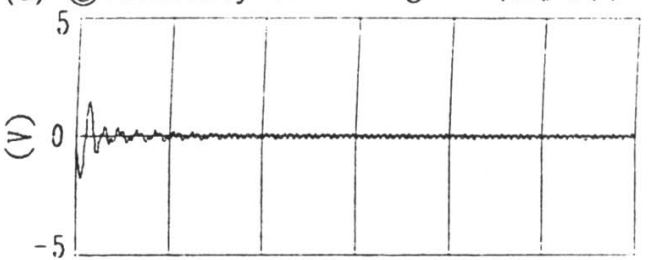

(f) (8): signal fed to a servo amplifier

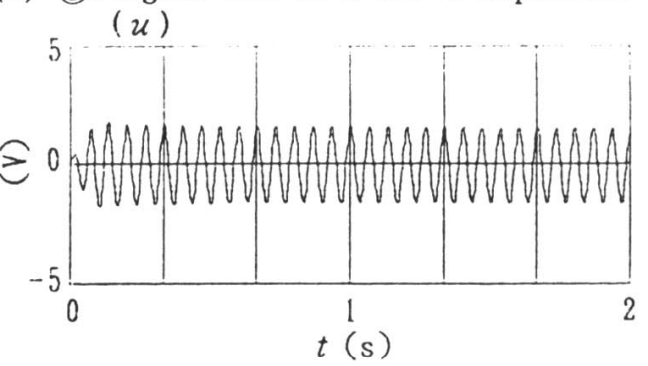

Fig.3.3 Simulated results of the adaptive control system
Fundamental circuit of the analog adaptive controller has been presented in Fig.3.2. To this fundamental circuit, some optional circuits were added. One of these optional circuits was a reset circuit of integral circuits. Switching a toggle button in the reset circuit, two condensers of integral circuits were shorted and integral circuits were initialized. Initializing integral circuits corresponded to setting gains $K_{1}$ and $K_{2}$ to those initial values $K_{10}$ and $K_{20}$. Switching the toggle button to the original position, integral circuits began to operate and calculations of the adaptive law started. In the beginning of experiments, integral circuits were initialized and sine or step signals were fed to the system as position demand signal. When the toggle button was

(a) position demand signal ( $r$ )

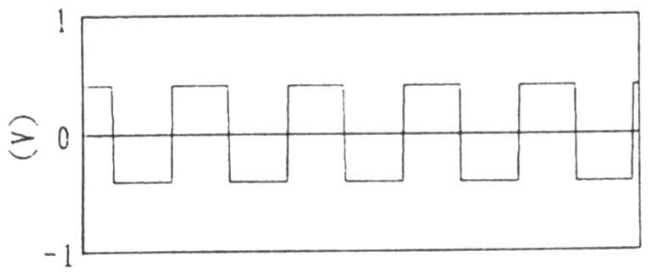

(b) load position signal $\left(K_{y} y\right)$

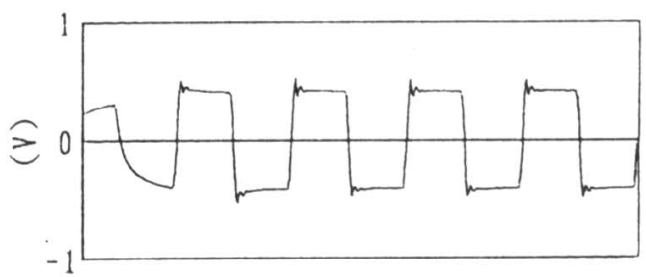

(c) load velocity signal $\left(K_{v} v\right)$

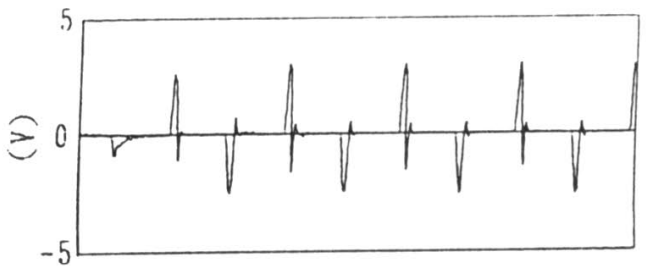

(d) reference model velocity signal $(K, v, n)$

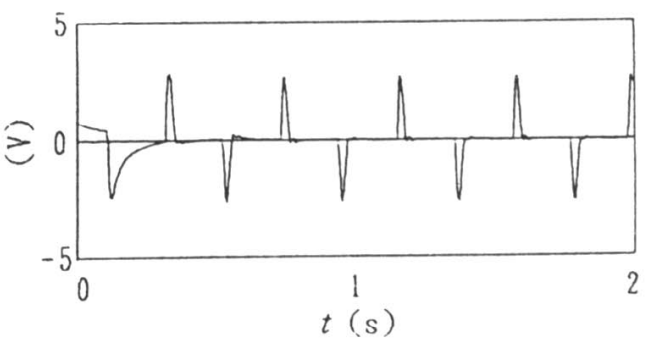

Fig.4.1 Experimental results of step responses 
switched, the adaptive control started. Transient signals of the system were recorded by a microcomputer NEC PC9801F with an A/D converter of 12 bit. Sampling interval of the $A / D$ was $5 \mathrm{~ms}$.

In Fig.4.1, experimental results of step responses of the adaptive control system are presented. In the beginning of

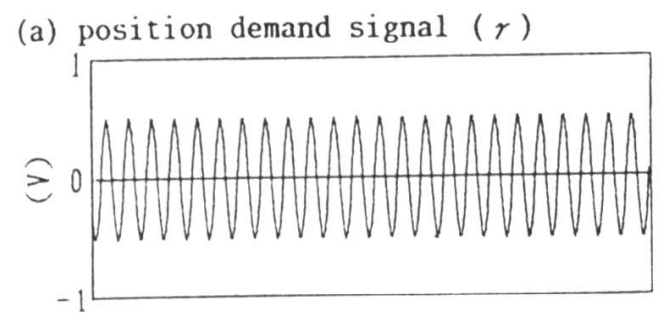

(b) load position signal ( $\left.K_{Y} y\right)$

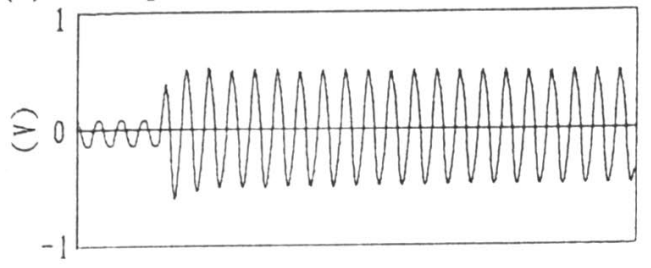

(c) load velocity signal $\left(K_{v} v\right)$

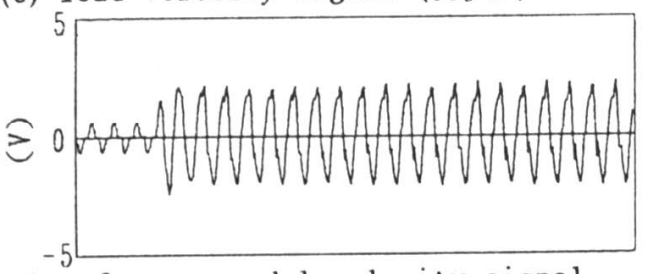

(d) reference model velocity signal

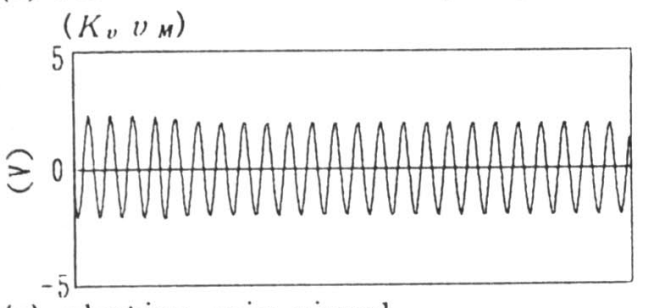

(e) adaptive gain signal

(corresponding to $K_{1}$ )

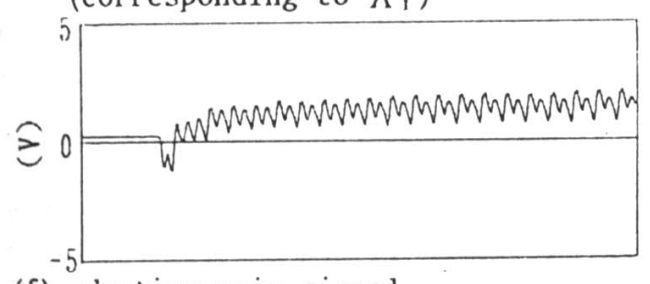

(f) adaptive gain signal

(corresponding to $K_{z}$ )

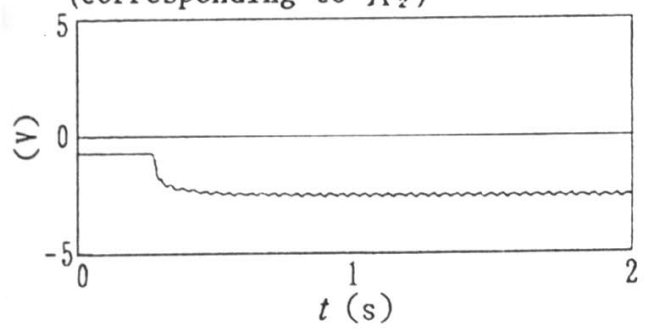

Fig.4.2 Experimental results of transient responses to sine input signal of $15 \mathrm{~Hz}$ experiments, integral circuits were initialized. After switching a toggle button and starting the adaptive control, the system showed considerably good step responses. Load velocity signal followed reference model velocity signal.

In Fig.4.2, experimental results of transient responses to sine input signal of $15 \mathrm{~Hz}$ are presented. In this figure, adaptive gain signals are also presented. Adaptive gain signals are signals (6) and (7) in Fig.3.2 (these signals correspond to $K_{1}$ and $K_{2}$ ). After start of the adaptive control, these adaptive gain signals began to change and showed stationary responses in $0.4 \mathrm{~s}$. Then load velocity signal followed reference model velocity signal. Since desired natural frequency $\omega_{n}$ was 170 rad/s

(a) position demand signal $(r)$

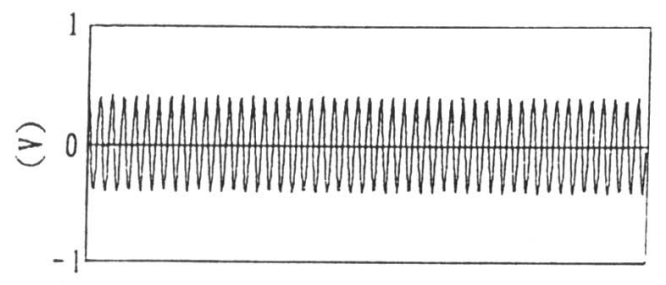

(b) load position signal $\left(K_{Y} y\right)$

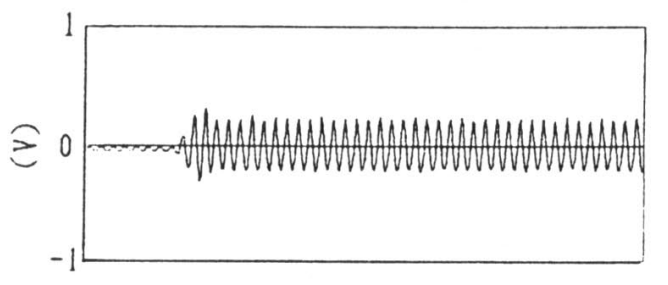

(c) load velocity signal $\left(K_{v} v\right)$

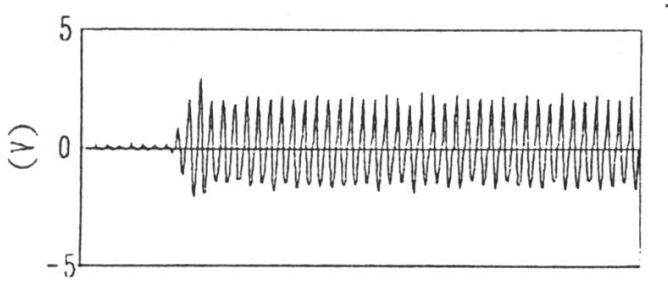

(d) reference model velocity signal

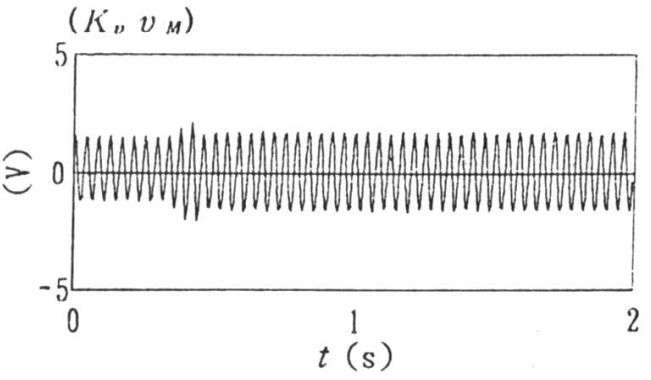

Fig.4.3 Experimental results of transient responses to sine input signal of $30 \mathrm{~Hz}$ 
$(27 \mathrm{~Hz})$ and desired damping ratio $\zeta$ was 0.5 , the adaptive control system was able to show good position responses to sine input signals of $15 \mathrm{~Hz}$. In experiments, transient responses of the system to sine input signals of $30 \mathrm{~Hz}$ were also tested. These experimental results are presented in Fig.4.3. Just after starting the adaptive control, the system showed stable and stationary responses. And load velocity signal followed reference model velocity signal. In experiments of the discrete-time adaptive control with a microcomputer using the same servo mechanism used in this study, the system was not able to show stable responses to such a relatively high frequency input signal. Since the computing time of the analog adaptive controller is theoretically zero, the continuous-time adaptive control system can show good responses to such a high frequency input signal.

\section{CONCLUSION}

In this study, an analog adaptive controller was investigated. At first, the adaptive law was constructed in continuous time description. Secondly, a CAD program for design of the controller was programed and an analog electronic circuit which realized the adaptive law was designed from various simulations. At last, experiments of the servo system with the analog adaptive controller were conducted. And it has been cleared that the analog adaptive control system showed stable and good responses to step input signals and sine input signals of relatively high frequency.

From investigation of band width of the analog adaptive controller, it is obvious that this controller can compute considerably higher frequency signals. Therefore investigation of the servo system consisted of a high speed oilhydraulic servomechanism and the analog adaptive controller is now being planned by the authors.

\section{REFERENCES}

(1) M.INOUE, K.TAKAHASHI and S.IKEO, "Application of the Model Reference Adaptive Control Theory to an Electrohydraulic Servosystem",J.of the Japan Hydraulics \& Pneumatics Soc., (in Japanese), Vol . 18, No.6, (1987), 467.

(2) K.TAKAHASHI, M.INOUE and S.IKEO, "Application of the Model Reference Adaptive Control Technique to an Electrohydraulic Servosystem", Proc. of ICFP, (1985), 68.
(3) K.YAMADA and Y.TANAKA, "A Study on Adaptive Control of an Electrohydraulic Servomechani sm", Proc.Hyd. Pneum.Symp.Fal1, (in Japanese), (1986), 51.

(4) K.SANADA, S.OHMURA, T.YAMASHITA, A.KITAGAiNA and T.TAKENAKA, "Improvement of Characteristics of Adaptive Control of an Electrohydraulic Servomechanism", Proc.Hyd.Pneum.Symp.-Spring, (in Japanese), (1987), 49.

(5) K.SANADA, A.KITAGAWA and Y.HAGIWARA, "A Study on an Electrohydraulic Servomechanism with an Analog Adaptive Controller",Proc.Hyd.Pneum.Symp.Spring, (in Japanese), (1988), 49.

(6) I.D.LANDOU and M.TOMIZUKA, Thoery \& Practice of Adaptive Control System, OHM Co.Ltd., (in Japanese), (1987).

(7) K.ICHIKAWA, K.KANAI, T.SUZUKI and H.TAMURA, Adaptive Control, SYOUSEIDO Co.Ltd., (in Japanese), (1984). 